International Mathematical Forum, 2, 2007, no. 28, 1375 - 1380

\title{
Strong Convergence of Nonexpansive Nonself-Mapping in Hilbert Space ${ }^{1}$
}

\author{
Rudong Chen and Zhichuan Zhu \\ Department of Mathematics \\ Tianjin Polytechnic University \\ Tianjin, 300160, P. R. China \\ chenrd@tjpu.edu.cn, zhuzcnh@yahoo.com.cn
}

\begin{abstract}
Let $C$ be a closed convex subset of a Hilbert space $H, T: C \rightarrow H$ a non-expansive nonself-mapping satisfying weakly inwardness condition such that $F(T) \neq \emptyset$. In this paper, we study the convergence of the following type sequence generated by

$$
x_{n+1}=P\left(\alpha_{n} f\left(x_{n}\right)+\left(1-\alpha_{n}\right) T x_{n}\right)
$$

where $P: H \rightarrow C$ is a projective operator, $f: C \rightarrow C$ is a fixed contractive mapping. we prove that the sequence $\left\{x_{n}\right\}$ converges strongly to a fixed point of $T$ under the weaker condition $T x_{n+1}-T x_{n} \rightarrow 0$, as $n \rightarrow \infty$. The result improves some recent results, especially Yisheng Song and Rudong Chen [5, J. Math. Anal. Appl., 321 (2006), 316-326] and Hongkun Xu [6, J. Math. Anal. Appl., 298 (2004), 279-291].
\end{abstract}

Keywords: Fixed point; nonexpansive nonself-mapping; strong convergence; Banach limit

\section{IntRoduCtion AND PRELIMINARIES}

Throughout the paper, let $H$ be a Hilbert space with inner product $\langle\cdot, \cdot\rangle$ and $\|\cdot\|$. We write $x_{n} \rightarrow x$ to indicate that the sequence $\left\{x_{n}\right\}$ converges weakly to $x$. Similarly, $x_{n} \rightarrow x$ will symbolize strong convergence. Let $C$ be a nonempty closed convex subset of $H$, and $T: C \rightarrow H$ be a non-expansive nonself-mapping such that $F(T) \neq \emptyset$. In 1992, Marino and Trombetta(see reference[3]) considered the contraction $S_{t}: C \rightarrow C$ and $U_{t}: C \rightarrow C$ defined by

$$
S_{t}(x)=t P T x+(1-t) u \quad x \in C
$$

\footnotetext{
${ }^{1}$ This work is supported by the National Science Foundation of China, Grant 10471033 and 10271011.
} 
and

$$
U_{t}(x)=P(t T x+(1-t) u) \quad x \in C
$$

for each $t \in[0,1)$ and each fixed $u \in C$, where $P: H \rightarrow C$ is a projective operator, then $S_{t}$ and $U_{t}$ has a unique fixed point. By Banach contractive theorem, there is a unique $x_{t} \in F\left(S_{t}\right), y_{t} \in F\left(U_{t}\right)$ such that

$$
x_{t}=t P T x_{t}+(1-t) u
$$

and

$$
y_{t}=P\left(t T y_{t}+(1-t) u\right)
$$

In 1995, $\mathrm{Xu}$ and Yin(see reference[2]) proved that if $T: C \rightarrow H$ is a nonexpansive nonself-mapping, then as $t \rightarrow 0$, the sequence $\left\{x_{t}\right\}$ defined by (1.1) strongly converges to $P_{F(T)} u$, the sequence $\left\{y_{t}\right\}$ defined by (1.2) strongly converges to $P_{F(T)} u$, where $P: H \rightarrow C$ is the nearest point projection of $H$ onto $C$, i.e. for each $x \in H, P x$ is the unique element of $C$ that satisfies $\|x-P x\|=d(x, C)=\inf _{c \in C}\|x-c\|$. This result which has been proved by Takahashi and Kim(see reference[4]) is true in Banach space too.

In 1992, Wittmann proved the following theorem(see reference[1]):

Let $H$ be a Hilbert space, $C$ be a nonempty closed convex subset of $H$, and $T: C \rightarrow C$ be a non-expansive mapping, and $F(T) \neq \phi$, the sequence $\left\{x_{n}\right\}$ defined by

$$
x_{n+1}=\alpha_{n} u+\left(1-\alpha_{n}\right) T x_{n} \quad \text { for } n=1,2,3 \cdots
$$

where $u \in C$ is a fixed point, $\left\{\alpha_{n}\right\} \in(0,1)$ and satisfies

$$
\lim _{n \rightarrow \infty} \alpha_{n}=0, \sum_{n=1}^{\infty}\left|\alpha_{n+1}-\alpha_{n}\right|<\infty, \text { and } \sum_{n=1}^{\infty} \alpha_{n}=\infty .
$$

Then $\left\{x_{n}\right\}$ strongly converges to $P u \in F(T)$, where $P: C \rightarrow F(T)$ is a projective operator.

Definition 1.1. $\mu$ is called a Banach limit if $\mu$ is a continuous linear functional on $l^{\infty}$ satisfying:

(i) $\|\mu(e)\|=1=\mu(1), e=(1,1,1 \cdots)$

(ii) $\mu\left(a_{n}\right)=\mu\left(a_{n+1}\right), \forall a_{n} \in\left(a_{0}, a_{1}, \cdots\right) \in l^{\infty}$

(iii) $\liminf \inf _{n \rightarrow \infty} a_{n} \leq \mu\left(a_{n}\right) \leq \limsup _{n \rightarrow \infty} a_{n}, \forall a_{n} \in\left(a_{0}, a_{1}, \cdots\right) \in l^{\infty}$

According to time and circumstances, we use $\mu\left(a_{n}\right)$ instead of $\mu\left(a_{0}, a_{1}, \cdots\right)$.

Further, we know the following result:

Lemma 1.2. (see reference [1]) For a given $a \in R$, for all $\left\{a_{n}\right\} \in l^{\infty}$ satisfies $\mu\left(a_{n}\right) \leq a$, if $\lim \sup _{n \rightarrow \infty}\left(a_{n+1}-a_{n}\right) \leq 0$, then $\limsup _{n \rightarrow \infty} a_{n} \leq a$.

The proof of the following Lemma is in [8]: 
Lemma 1.3. Let $\left\{\alpha_{n}\right\}$ be a sequence of nonnegative real numbers satisfying the property

$$
\alpha_{n+1} \leq\left(1-\gamma_{n}\right) \alpha_{n}+\delta_{n}, \forall n \geq 0
$$

where $\left\{\gamma_{n}\right\} \in(0,1), \sum_{n=0}^{\infty}\left|\delta_{n}\right|<\infty$ and satisfies $\sum_{n=0}^{\infty} \gamma_{n}=\infty$, $\lim \sup _{n \rightarrow \infty} \frac{\delta_{n}}{\gamma_{n}} \leq$ 0 .

Then $\lim _{n \rightarrow \infty} \alpha_{n}=0$.

We now define

$$
U_{t} x=P(t f(x)+(1-t) T x), \forall x \in C,
$$

where $P: H \rightarrow C$ is a projective operator and $f: C \rightarrow C$, for some $\beta \in(0,1)$ is a fixed contractive mapping i.e. $\forall x, y \in C$ such that $\|f(x)-f(y)\| \leq$ $\beta\|x-y\|$. By Banach contractive theorem, there is a unique $x_{t} \in C$ such that

$$
\left.x_{t}=P\left(t f\left(x_{t}\right)+(1-t) T x_{t}\right)\right)
$$

We also can define the following explicit iterative sequence:

$$
x_{n+1}=P\left[\alpha_{n} f\left(x_{n}\right)+\left(1-\alpha_{n}\right) T x_{n}\right], n=1,2, \cdots
$$

where $\alpha_{n} \in(0,1)$.

\section{Main Results}

Recall that a nonself-mapping $T: C \rightarrow H$ is said to satisfy the inwardness condition if $T x \in I_{C}(x)$ for all $x \in C$, where $I_{C}(x)$ is the inward set relative to $C$ given by:

$$
I_{C}(x)=\{x+\lambda(y-z): y \in C, \lambda \geq 0\},
$$

and respectively to satisfy the weak inwardness condition if $T x \in \overline{I_{C}(x)}$ for all $x \in C$, where $\overline{I_{C}(x)}$ is the closure of $I_{C}(x)$ in the norm topology.

Lemma 2.1. ([5], Theorem2.2)Let $H$ be a Hilbert space, $C$ be a nonempty closed convex subset of $H$. T $: C \rightarrow H$ is a nonexpansive mapping from $C$ into $H$ satisfying the weak inwardness condition and $F(T) \neq \emptyset, f: C \rightarrow C$ is a fixed contractive mapping, and the sequence $\left\{x_{t}\right\}$ is defined by (1.3), where $P$ is a nonexpansive retract of $H$ onto $C$. As $t \rightarrow 0^{+}$, then $\left\{x_{t}\right\}$ converges strongly to some fixed point $u$ of $T$ such that $u$ is the unique solution in $F(T)$ to the following variational inequality:

$$
\langle(I-f) u, j(u-p)\rangle \leq 0, \quad \text { for all } p \in F(T) .
$$

From lemma 2.1 we have the following results directly:

$$
\begin{aligned}
& (i) T x_{t} \rightarrow u, t \rightarrow 0^{+} \\
& (i i) t f\left(x_{t}\right)+(1-t) T x_{t}-x_{t} \rightarrow 0, t \rightarrow 0^{+}
\end{aligned}
$$


Theorem 2.2. Let $C$ be a closed convex subset of a Hilbert space $H, T$ : $C \rightarrow H$ be a nonexpansive mapping satisfying weakly inwardness condition, and $F(T) \neq \phi$, Let $f: C \rightarrow C$ is a fixed contractive mapping. The sequence $\left\{x_{n}\right\}$ is defined by (1.4) i.e.

$$
x_{n+1}=P\left[\alpha_{n} f\left(x_{n}\right)+\left(1-\alpha_{n}\right) T x_{n}\right], n=1,2, \ldots
$$

where $P: H \rightarrow C$ is projective operator and $\alpha_{n} \in(0,1)$ satisfies

$$
\lim _{n \rightarrow \infty} \alpha_{n}=0, \text { and }, \sum_{n=1}^{\infty} \alpha_{n}=\infty .
$$

If $T x_{n+1}-T x_{n} \rightarrow 0$ as $n \rightarrow \infty$, then $\left\{x_{n}\right\}$ strongly converges to $u=\lim _{t \rightarrow 0} x_{t} \in$ $F(T)$, where $\left\{x_{t}\right\}$ is defined by (1.3).

Proof. First we show $\left\{x_{n}\right\}$ is bounded. Let $q \in F(T)$, then

$$
\begin{aligned}
\left\|x_{n+1}-q\right\| & =\left\|P\left[\alpha_{n} f\left(x_{n}\right)+\left(1-\alpha_{n}\right) T x_{n}\right]-P q\right\| \\
& \leq\left\|\alpha_{n}\left(f\left(x_{n}\right)-q\right)+\left(1-\alpha_{n}\right)\left(x_{n}-q\right)\right\| \\
& \leq \alpha_{n}\left(\left\|f\left(x_{n}\right)-f(q)\right\|+\|f(q)-q\|\right)+\left(1-\alpha_{n}\right)\left\|x_{n}-q\right\| \\
& \leq\left(1-(1-\beta) \alpha_{n}\right)\left\|x_{n}-q\right\|+\alpha_{n}\|f(q)-q\| \\
& \leq \max \left\{\left\|x_{n}-q\right\|, \frac{1}{1-\beta}\|f(q)-q\|\right\},
\end{aligned}
$$

By induction, $\left\|x_{n}-q\right\| \leq \max \left\{\left\|x_{0}-q\right\|, \frac{1}{1-\beta}\|f(q)-q\|\right\}, n \geq 0$, so we have $\left\{x_{n}\right\}$ is bounded, and so are $\left\{f\left(x_{n}\right)\right\}$ and $\left\{T x_{n}\right\}$.

1. $\mu_{n}\left\langle f(u)-u, T x_{n}-u\right\rangle \leq 0$ Indeed

$$
\begin{aligned}
\left\|x_{n+1}-P T x_{n}\right\| & =\left\|P\left[\alpha_{n} f\left(x_{n}\right)+\left(1-\alpha_{n}\right) T x_{n}\right]-P T x_{n}\right\| \\
& \leq\left\|\alpha_{n} f\left(x_{n}\right)+\left(1-\alpha_{n}\right) T x_{n}-T x_{n}\right\| \\
& \leq \alpha_{n}\left\|f\left(x_{n}\right)-T x_{n}\right\|
\end{aligned}
$$

Since $\lim _{n \rightarrow \infty} \alpha_{n}=0$, so we have $\left\|x_{n+1}-P T x_{n}\right\| \rightarrow 0$ as $n \rightarrow \infty$. Noting that in the Hilbert space $H$, there holds that the inequality $\|x+y\|^{2} \leq\|x\|^{2}+$ $2\langle y, x+y\rangle$, for all $x, y \in H$, then we have

$$
\begin{aligned}
\left\|x_{t}-x_{n+1}\right\|^{2} & =\left\|x_{t}-P T x_{n}+P T x_{n}-x_{n+1}\right\|^{2} \\
& \leq\left\|x_{t}-P T x_{n}\right\|^{2}+2\left\langle P T x_{n}-x_{n+1}, x_{t}-x_{n+1}\right\rangle \\
& \leq\left\|P\left[t f\left(x_{t}\right)+(1-t) T x_{t}\right]-P T x_{n}\right\|^{2} \\
& +2\left\|P T x_{n}-x_{n+1}\right\|\left\|x_{t}-x_{n+1}\right\| \\
& \leq\left\|t f\left(x_{t}\right)+(1-t) T x_{t}-T x_{n}\right\|^{2} \\
& +2\left\|P T x_{n}-x_{n+1}\right\|\left\|x_{t}-x_{n+1}\right\|
\end{aligned}
$$


In the above inequality we set $y_{t}=t f\left(x_{t}\right)+(1-t) T x_{t}$, then

$$
\begin{aligned}
\left\|t f\left(x_{t}\right)+(1-t) T x_{t}-T x_{n}\right\|^{2} & =\left\|t\left(f\left(x_{t}\right)-T x_{n}\right)+(1-t)\left(T x_{t}-T x_{n}\right)\right\|^{2} \\
& \leq(1-t)^{2}\left\|T x_{t}-T x_{n}\right\|^{2} \\
& +2 t\left\langle f\left(x_{t}\right)-T x_{n}, y_{t}-T x_{n}\right\rangle \\
& =(1-t)^{2}\left\|T x_{t}-T x_{n}\right\|^{2} \\
& +2 t\left\langle f\left(x_{t}\right)-T x_{n}, y_{t}-T x_{t}+T x_{t}-T x_{n}\right\rangle \\
& =(1-t)^{2}\left\|T x_{t}-T x_{n}\right\|^{2} \\
& +2 t\left\langle f\left(x_{t}\right)-T x_{n}, y_{t}-T x_{t}\right\rangle \\
& +2 t\left\langle f\left(x_{t}\right)-T x_{t}, T x_{t}-T x_{n}\right\rangle \\
& +2 t\left\langle T x_{t}-T x_{n}, T x_{t}-T x_{n}\right\rangle \\
& \leq\left(1+t^{2}\right)\left\|x_{t}-x_{n}\right\|^{2} \\
& +2 t\left\langle f\left(x_{t}\right)-T x_{n}, y_{t}-T x_{t}\right\rangle \\
& +2 t\left\langle f\left(x_{t}\right)-T x_{t}, T x_{t}-T x_{n}\right\rangle
\end{aligned}
$$

From $(2.1)(2.2)$ we can get

$$
\begin{aligned}
2 t\left\langle f\left(x_{t}\right)-T x_{t}, T x_{n}-T x_{t}\right\rangle & \leq\left(1+t^{2}\right)\left\|x_{t}-x_{n}\right\|^{2}+2 t\left\langle f\left(x_{t}\right)-T x_{n}, y_{t}-T x_{t}\right\rangle \\
& +2\left\|P T x_{n}-x_{n+1}\right\|\left\|x_{t}-x_{n+1}\right\|-\left\|x_{t}-x_{n+1}\right\|^{2}
\end{aligned}
$$

Now we apply Banach limit to the above inequality,

$$
\begin{aligned}
2 t \mu\left\langle f\left(x_{t}\right)-T x_{t}, T x_{n}-T x_{t}\right\rangle & \leq\left(1+t^{2}\right) \mu\left(\left\|x_{t}-x_{n}\right\|^{2}\right)+2 t \mu\left\langle f\left(x_{t}\right)-T x_{n}, y_{t}-T x_{t}\right\rangle \\
& -\mu\left(\left\|x_{t}-x_{n+1}\right\|^{2}\right) \\
& =t^{2} \mu\left(\left\|x_{t}-x_{n}\right\|^{2}\right)+2 t \mu\left\langle f\left(x_{t}\right)-T x_{n}, y_{t}-T x_{t}\right\rangle
\end{aligned}
$$

i.e.

$$
\mu\left\langle f\left(x_{t}\right)-T x_{t}, T x_{n}-T x_{t}\right\rangle \leq \frac{t}{2} \mu\left(\left\|x_{t}-x_{n}\right\|^{2}\right)+\mu\left\langle f\left(x_{t}\right)-T x_{n}, y_{t}-T x_{t}\right\rangle
$$

So we have $\lim \sup _{t \rightarrow 0} \mu\left\langle f\left(x_{t}\right)-T x_{t}, T x_{n}-T x_{t}\right\rangle \leq 0$, i.e.

$$
\mu\left\langle f(u)-u, T x_{n}-u\right\rangle \leq 0 .
$$

2. we shall prove $\lim \sup _{n \rightarrow \infty}\left\langle f(u)-u, T x_{n}-u\right\rangle \leq 0$.

We set $a_{n}=\left\langle f(u)-u, T x_{n}-u\right\rangle$, since $\mu\left(a_{n}\right) \leq 0$ for all Banach limit and $\left\{T x_{n}\right\}$ is bounded, now we take the subsequence $\left\{T x_{n_{j}}\right\}$ of $\left\{T x_{n}\right\}$, such that $\limsup \sup _{n \rightarrow 0}\left(a_{n+1}-a_{n}\right)=\lim _{j \rightarrow \infty}\left(a_{n_{j}+1}-a_{n_{j}}\right)$, from $T x_{n_{j}+1}-T x_{n_{j}} \rightarrow 0$, as $j \rightarrow \infty$, then

$$
\begin{aligned}
\limsup \sup _{n \rightarrow \infty}\left(a_{n+1}-a_{n}\right) & =\lim _{j \rightarrow \infty}\left(a_{n_{j}+1}-a_{n_{j}}\right) \\
& =\lim _{j \rightarrow \infty}\left\langle f(u)-u, T x_{n_{j}+1}-T x_{n_{j}}\right\rangle=0
\end{aligned}
$$

From lemma1.2 we have

$$
\limsup _{n \rightarrow \infty} a_{n} \leq 0
$$

i.e.

$$
\limsup _{n \rightarrow \infty}\left\langle f(u)-u, T x_{n}-u\right\rangle \leq 0 .
$$

Let $\theta_{n}=\max \left\{\left\langle f(u)-u, T x_{n}-u\right\rangle, 0\right\}$, then $\theta_{n} \rightarrow 0$ as $n \rightarrow \infty$. 
3. Finally we prove $x_{n} \rightarrow u$ as $n \rightarrow \infty$.

$$
\begin{aligned}
\left\|x_{n+1}-u\right\|^{2} & =\left\|P\left[\alpha_{n} f\left(x_{n}\right)+\left(1-\alpha_{n}\right) T x_{n}\right]-u\right\|^{2} \\
& \leq\left\|\alpha_{n}\left(f\left(x_{n}\right)-u\right)+\left(1-\alpha_{n}\right)\left(T x_{n}-u\right)\right\|^{2} \\
& =\alpha_{n}^{2}\left\|f\left(x_{n}-u\right)\right\|^{2}+\left(1-\alpha_{n}\right)^{2}\left\|T x_{n}-u\right\|^{2} \\
& +2 \alpha_{n}\left(1-\alpha_{n}\right)\left\langle f\left(x_{n}\right)-u, T x_{n}-u\right\rangle \\
& \leq\left(1-\alpha_{n}\right)^{2}\left\|x_{n}-u\right\|^{2}+\alpha_{n}^{2}\left\|f\left(x_{n}\right)-u\right\|^{2} \\
& +2 \alpha_{n}\left(1-\alpha_{n}\right)\left(\left\langle f\left(x_{n}\right)-f(u), T x_{n}-u\right\rangle+\left\langle f(u)-u, T x_{n}-u\right\rangle\right) \\
& \leq\left(1-\alpha_{n}\right)^{2}\left\|x_{n}-u\right\|^{2}+\alpha_{n}^{2}\left\|f\left(x_{n}\right)-u\right\|^{2}+2 \alpha_{n}\left(1-\alpha_{n}\right) \beta\left\|x_{n}-u\right\|^{2} \\
& +2 \alpha_{n}\left(1-\alpha_{n}\right)\left\langle f(u)-u, T x_{n}-u\right\rangle \\
& \leq\left[\left(1-2 \alpha_{n}+\alpha_{n}^{2}+2 \alpha_{n}\left(1-\alpha_{n}\right) \beta\right]\left\|x_{n}-u\right\|^{2}+\alpha_{n}\left(\left\|f\left(x_{n}\right)-u\right\|^{2}\right.\right. \\
& \left.+2\left(1-\alpha_{n}\right)\left\langle f(u)-u, T x_{n}-u\right\rangle\right] \\
& =\left(1-\alpha_{n}^{*}\right)\left\|x_{n}-u\right\|^{2}+\alpha_{n}^{*} \beta_{n}^{*},
\end{aligned}
$$

where

$$
\begin{gathered}
\alpha_{n}^{*}=\alpha_{n}\left(2-\alpha_{n}-2 \beta\left(1-\alpha_{n}\right)\right) \\
\beta_{n}^{*}=\frac{2\left(1-\alpha_{n}\right)\left\langle f(u)-u, T x_{n}-u\right\rangle+\alpha_{n}\left\|f\left(x_{n}\right)-u\right\|^{2}}{2-2 \beta\left(1-\alpha_{n}\right)}
\end{gathered}
$$

Noting that $\lim _{n \rightarrow \infty} \alpha_{n}^{*}=0, \sum_{n=0}^{\infty} \alpha_{n}^{*}=\infty$ and $\limsup _{n \rightarrow} \beta_{n}^{*} \leq 0$.

From lemma1.3 we have

$$
x_{n} \rightarrow u, \text { as } n \rightarrow \infty
$$

The proof is complete.

\section{REFERENCES}

1. R. Witmann, approximation of fixed points of nonexpansive mappings, Archs. Math., $\mathbf{5 8}$ (1992), 486-491.

2. H.K. Xu and X.M. Yin, Srong convergence theorems for nonexpansive nonself-mappings, Nonlinear Anal., 24 (1995), 223-228.

3. G. Marino and G. Trombetta, On approximating fixed points, Indian J. Anal., 34 (1992), 91-98.

4. W. Takahashi and G.E. Kim, Srong convergence of approximants to fixed points of nonexpansive nonself-mappings in Banach space, Nonlinear Anal. 32(1998)447-454.

5. Yisheng Song and Rudong Chen, Viscosity approximation method for nonexpansive nonself-mappings, J. Math. Anal. Appl. 321 (2006), 316-326.

6. H.K. Xu, Viscosity approximation methods for nonexpansive mappings, J. Math. Anal. Appl., 298 (2004), 279-291.

\section{Received: August 30, 2006}

